\title{
Recent research directions in Fribourg: nuclear dynamics in resonances revealed by 2-dimensional EEL spectra, electron collisions with ionic liquids and electronic excitation of pyrimidine ${ }^{\star}$
}

\author{
Michael Allan ${ }^{1, a}$, Khrystyna Regeta ${ }^{1}$, Jimena D. Gorfinkiel ${ }^{2}$, Zdeněk Mašín $^{3}$, \\ Stefan Grimme ${ }^{4}$, and Christoph Bannwarth ${ }^{4}$ \\ 1 Department of Chemistry, University of Fribourg, chemin du Musée 9, 1700 Fribourg, Switzerland \\ 2 Department of Physical Sciences, The Open University, Walton Hall, Milton Keynes, MK7 6AA, UK \\ 3 Max-Born Institute for Nonlinear Optics and Short Pulse Spectroscopy, Max-Born-Strasse 2A, 12489 Berlin, Germany \\ 4 Mulliken Center for Theoretical Chemistry, Institut für Physikalische und Theoretische Chemie, Universität Bonn, \\ Beringstrasse 4, 53115 Bonn, Germany
}

Received 29 February 2016 / Received in final form 12 April 2016

Published online 26 May 2016 - (c) EDP Sciences, Società Italiana di Fisica, Springer-Verlag 2016

\begin{abstract}
The article briefly reviews three subjects recently investigated in Fribourg: (i) electron collisions with surfaces of ionic liquids, (ii) two-dimensional (2D) electron energy loss spectra and (iii) resonances in absolute cross sections for electronic excitation of unsaturated compounds. Electron energy loss spectra of four ionic liquids revealed a number of excited states, including triplet states. A solution of a dye in an ionic liquid showed an energy-loss band of the solute, but not in all ionic liquids. 2D spectra reveal state-to-state information (given resonance to given final state) and are shown to be an interesting means to gain insight into dynamics of nuclear motion in resonances. Absolute cross sections for pyrimidine are reported as a function of scattering angle and as a function of electron energy. They reveal resonant structure which was reproduced very nicely by R-matrix calculations. The calculation provided an assignment of the resonances which reveals common patterns in compounds containing double bonds.
\end{abstract}

\section{Introduction}

The article is inspired by the POSMOL 2015 talk and will briefly review three subjects recently investigated in Fribourg: (i) electron collisions with surfaces of ionic liquids, (ii) two-dimensional electron energy loss spectra and (iii) resonances in absolute cross sections for electronic excitation of unsaturated compounds.

\section{Experiment}

The measurements were performed with the Fribourg electron energy loss spectrometer [1-3] shown schematically in Figure 1. It is a conventional hemispherical analyzer instrument but careful design of the electron optics, choice of materials, differential pumping and fast pumps (two 6 inch and one 16 inch diffusion pumps) permit it to

\footnotetext{
* Contribution to the Topical Issue "Advances in Positron and Electron Scattering", edited by Paulo Limao-Vieira, Gustavo Garcia, E. Krishnakumar, James Sullivan, Hajime Tanuma and Zoran Petrovic.

a e-mail: michael.allan@unifr.ch
}

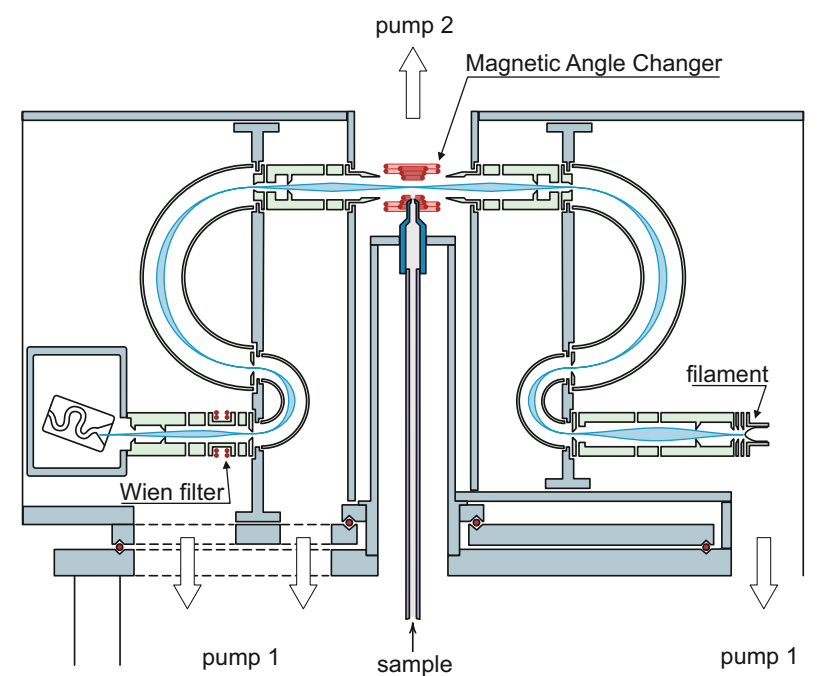

Fig. 1. Scheme of the spectrometer.

reach low energies (about $50 \mathrm{meV}$ for incident electrons, about $25 \mathrm{meV}$ for scattered electrons) and low background (down to $0.1 \mathrm{c} / \mathrm{s}$, depending on electron energy and scattering angle). The design relied on early tools for electron 


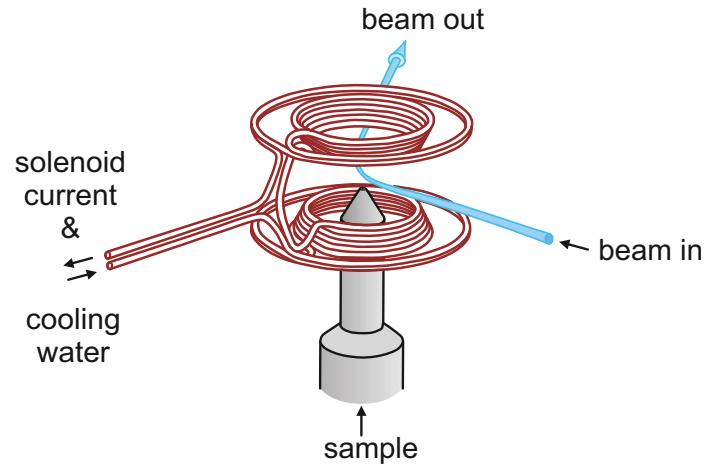

Fig. 2. Magnetic Angle Changer.

optics simulation - the matrix method with thick lens formalism, parametrized by Harting and Read [4]. The instrument has later been equipped with a magnetic angle changer (MAC) [5] - an ingenious device invented by Read et al. $[6,7]$. The powerful charged particle optics (CPO) program [8] written by Frank H. Read was available in the meantime and was essential for the design of the MAC device. The Fribourg version avoids a bulky solenoid structure (Fig. 2) to minimize interference with the flow of sample gas. An elaborate protocol has been applied to optimize and quantify the instrumental response function (sensitivity) as a function of the residual and incident electron energies and the scattering angle and to correct cross sections for it $[1,3,9]$.

\section{Ionic liquids}

At the last moment, with my retirement rapidly approaching, I (MA) fulfilled my long time dream to study electron collisions with a liquid surface [10]. For this purpose a small molybdenum wire loop with a droplet of an ionic liquid (IL) was installed in the collision region as shown in Figure 3. The wire loop is movable and can be withdrawn from, and returned to, the collision region using a stepping motor and a rotary feed-through. This was essential because the instrument must be periodically "tuned" (i.e., adjusted for drifts), and the correctness of its response function verified, on helium target in the course of the measurements using the protocol developed for gas phase studies.

Ionic liquids, besides being a fascinating and popular class of compounds by themselves, offer obvious advantages for electron spectroscopy work: they have very low volatility making them compatible with high vacuum and they are conductive, preventing build up of surface charge. There was existing electron spectroscopy work on ionic liquids as detailed in our publication [10]. These include photoelectron spectroscopy, penning ionization with metastable helium atoms, high energy electron energy loss spectroscopy in electron microscopes, and vibrational electron energy loss. There is, of course, extended amount of literature on electron collision with solid surfaces, which is also closely related with the present work.

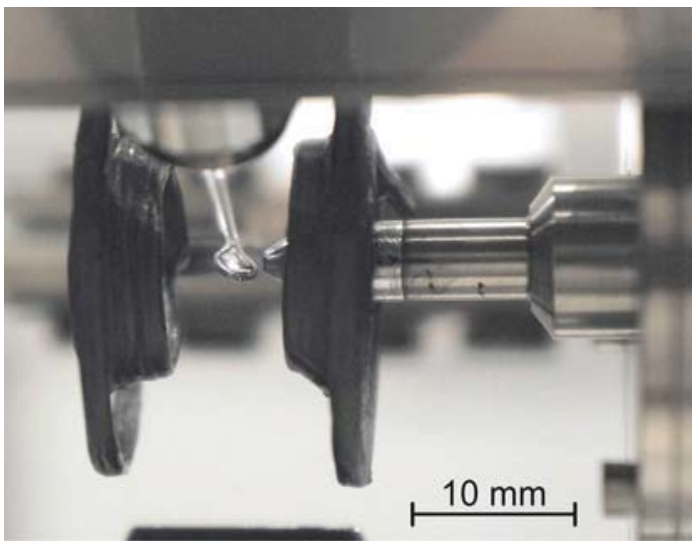

Fig. 3. A droplet of an ionic liquid suspended in the Electron Energy Loss Spectrometer. The electron beam arrives vertically from the top and scattered electrons are collected at $135^{\circ}$ with respect to the direction of the incident beam.

The first point of worry was whether the ionic liquid droplet will not be strongly contaminated by pump oil and other residual gases in a chamber with a residual pressure of $10^{-7}$ mbar. This question was addressed by (i) recording the vibrational energy loss spectrum of the ionic liquid and comparing it with the infrared spectrum, (ii) recording energy loss spectra of four different ionic liquids and (iii) monitoring possible changes in the spectra over several days. The conclusion was that our energy loss spectra are due exclusively to the ionic liquid and, perhaps somewhat surprisingly, that there is no evidence of signals due to contaminants. Our "plan B" was to use dropping ionic liquid target (inspired by the dropping mercury electrode invented by Kučera [11] and used by Heyrovský for polarography [12]) to provide a constantly fresh surface but this was finally not necessary.

The second point of worry was that the spectra will be entirely "washed out" by multiple collisions, preventing any detailed interpretation. It will be shown below that this aspect can be successfully minimized by working at the specular angle and at a (residual) electron energy of $10 \mathrm{eV}$ or higher.

The two exciting classes of phenomena in electron collisions are: (i) study of excited states of the target by recording electron energy loss spectra, with emphasis on forbidden transitions and highly excited states, and (ii) study of resonances by recording "energy dependence spectra" where a given excitation process is measured as a function of the incident electron energy. An additional exciting aspect resides in the short penetration depth of slow electrons (about 5-10 $\AA 13,14]$ ), permitting the study of surface structure.

\subsection{Energy-loss spectra}

As shown in our more specialized paper [10], electron energy loss spectra recorded near threshold, by collecting 0-2 eV electrons, have strong continuous background, assigned to excitation of a quasi-continuum of high overtones 


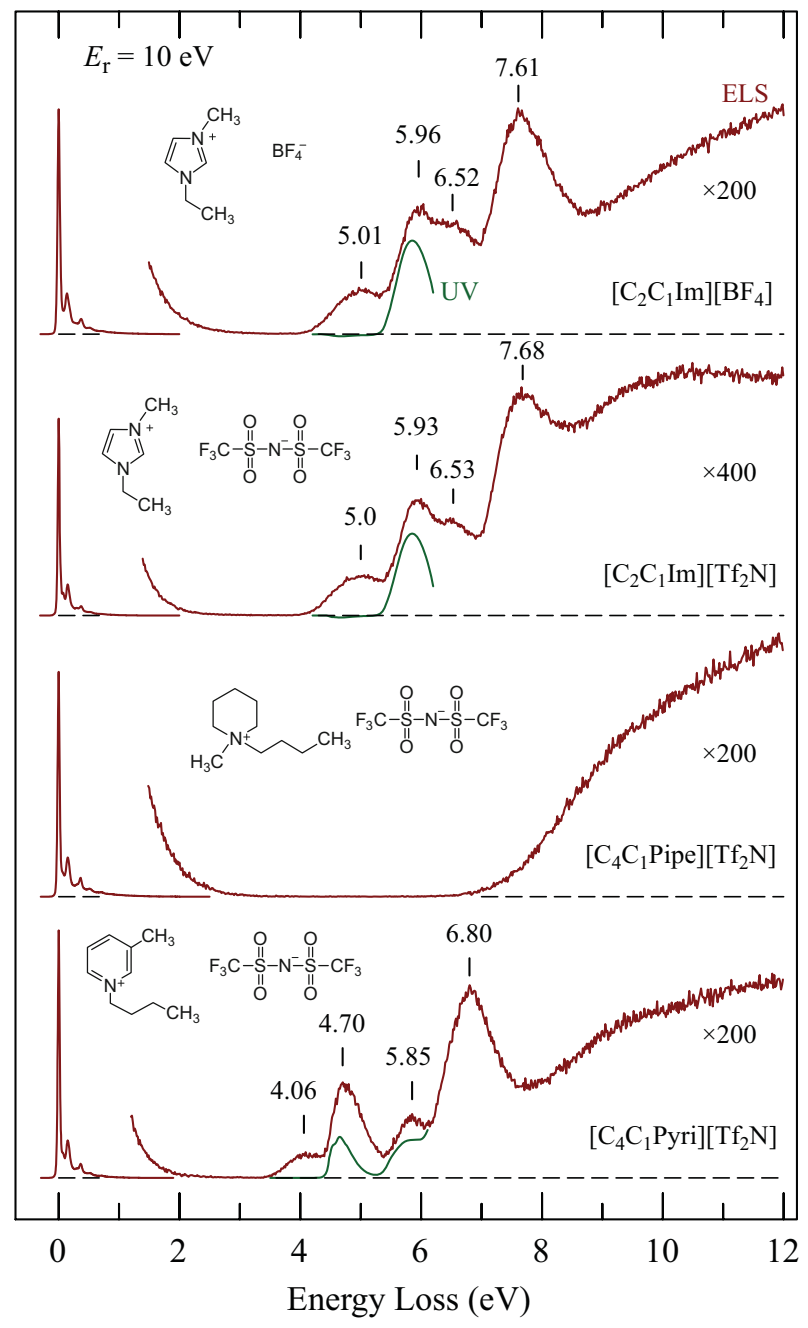

Fig. 4. Red lines: energy loss spectra recorded at a residual electron energy of $10 \mathrm{eV}$; green lines: conventional ultraviolet spectra of diluted methanol solutions (from Ref. [10]).

and combination vibrations of low-frequency modes. The mechanisms could be multiple collisions which necessarily lead to slow electrons, and/or the "unspecific" vibrational excitation whereby resonances excite a continuum of high vibrational levels at and near threshold in large molecules [15]. These two mechanisms are less active in spectra recorded by collecting faster electrons, about $10 \mathrm{eV}$ and above, such as shown in Figure 4, which show predominantly discrete vibrational and electronic bands.

Certain conclusions can be reached already on a qualitative level. The absence of the lowest energy loss bands in the UV spectra suggests that they arise from triplet states. The narrow bands in the $3.5-8 \mathrm{eV}$ spectral region must be due to the $\pi$-systems of the cations because they are absent in $\left[\mathrm{C}_{4} \mathrm{C}_{1}\right.$ Pyri $]\left[\mathrm{Tf}_{2} \mathrm{~N}\right]$, the IL without such a system (second spectrum from the bottom in Fig. 4).

A deeper insight into the nature of the excited states was obtained with the help of density functional theory multireference configuration interaction (DFT/MRCI) calculations - an example of the results is shown in Figure 5. The agreement is very good for both the singlet

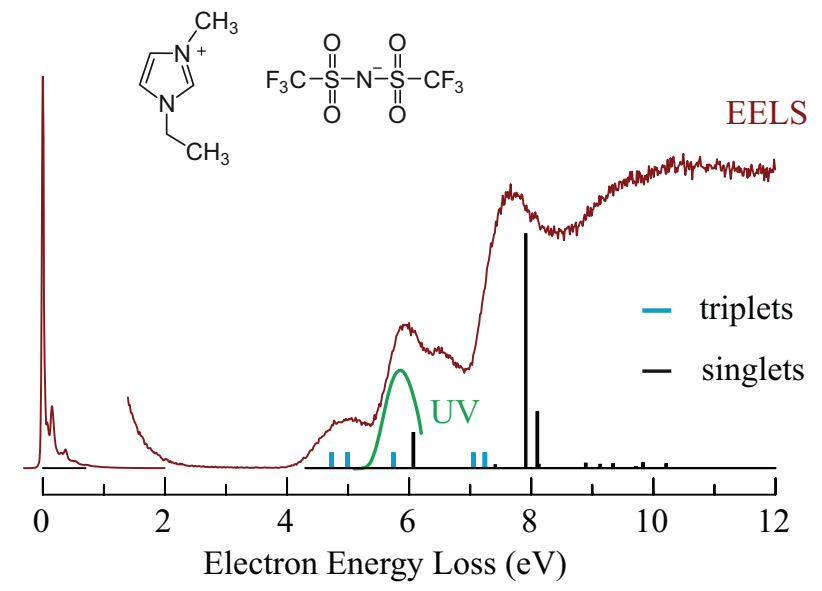

Fig. 5. Red line: energy loss spectrum recorded at a residual electron energy of $10 \mathrm{eV}$; vertical lines: results of DFT/MRCI calculations. The heights of the black lines reflect the calculated oscillator strengths (adopted from Ref. [10]).

and triplet states. The calculation provided a detailed assignment of the states and confirmed that the bands in the $3.5-8 \mathrm{eV}$ region are due to $\pi, \pi^{*}$ states of the cations [10]. The calculations have also shown that the very broad bands above $8 \mathrm{eV}$ are due to transitions of the anions. The calculations have further emphasized the importance of the correct choice of the model - models with low amount of Fock exchange (e.g., in B3LYP) generate artifact charge-transfer (CT) states. A rangeseparated functional or hybrids with about $50 \%$ Fock exchange are necessary to properly describe CT states in such systems [16]. (More details on the problems linked to the study of CT states with semi-local density functionals can be found in Ref. [17].)

\subsection{EEL spectra of solutions}

EELS of concentrated (1 mol/L) solutions of methylene green (MG) dye in two ionic liquids were recorded and are compared to the EELS of the neat ionic liquids in Figure 6. UV spectrum of MG solution in methanol is also shown for comparison. The surprising observation is that whereas the MG band clearly appears in the lower panel of Figure 6, it is absent in the upper panel, although the two solutions were equally concentrated. The likely explanation is that the longer alkyl chains of the ionic liquid in the upper panel aggregate to form a hydrophobic layer on the surface of the $\left[\mathrm{C}_{4} \mathrm{C}_{1}\right.$ Pyri $]\left[\mathrm{Tf}_{2} \mathrm{~N}\right]$ liquid and that the ionic MG dye is expelled from this layer and is consequently not reachable in view of the short penetration depth of the electrons. These observations indicate that ILs may be used as a host to study nonvolatile compounds by EELS and that information on surface structure of the solution is obtained.

The spectrum of MG started to disappear after several hours of exposure to the electron beam, indicating a chemical transformation induced by electrons. 


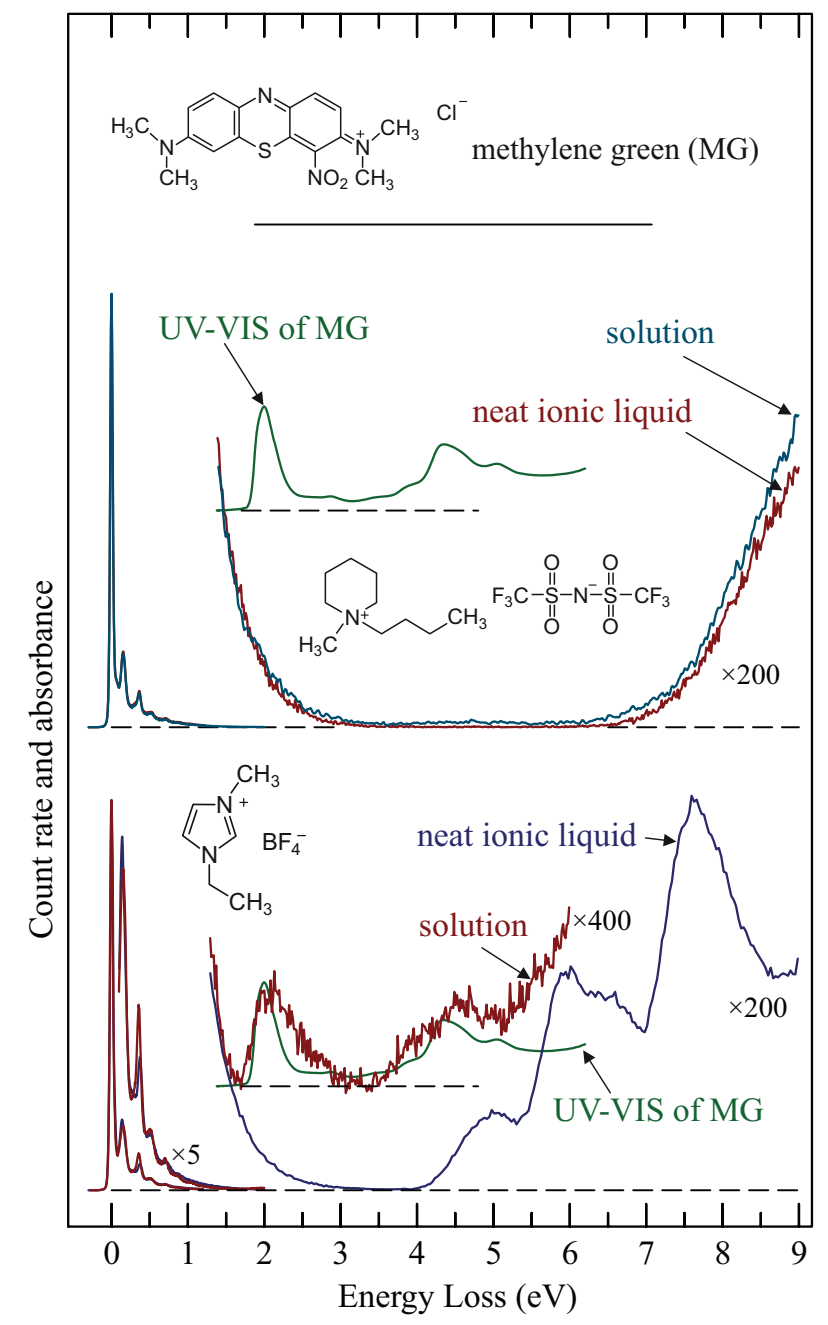

Fig. 6. EELS of solutions of methylene green in two ionic liquid, compared to EELS of the neat ionic liquids. UV spectra of strongly diluted methanol solutions of methylene green are also shown for comparison.

\subsection{Resonances in ionic liquids}

The signal for various vibrational and electronic excitation processes was recorded as a function of electron energy but the data was entirely flat - there were no bands which could be assigned as resonances. An indirect indication of resonant vibrational excitation is given by the enhanced (compared to IR) intensity of vibrational overtones. It appears that any such resonances are extremely broadened and do not give rise to bands.

\section{2D EEL spectra as a tool to study nuclear dynamics in resonances}

2D electron collision spectra contain rich information about the motion of a nuclear wave packet on a resonant potential surface - a key ingredient to understanding electron-induced chemistry. It is possible because the motion of the nuclear wave packet is characterized by a competition between propagation and detachment, the wave

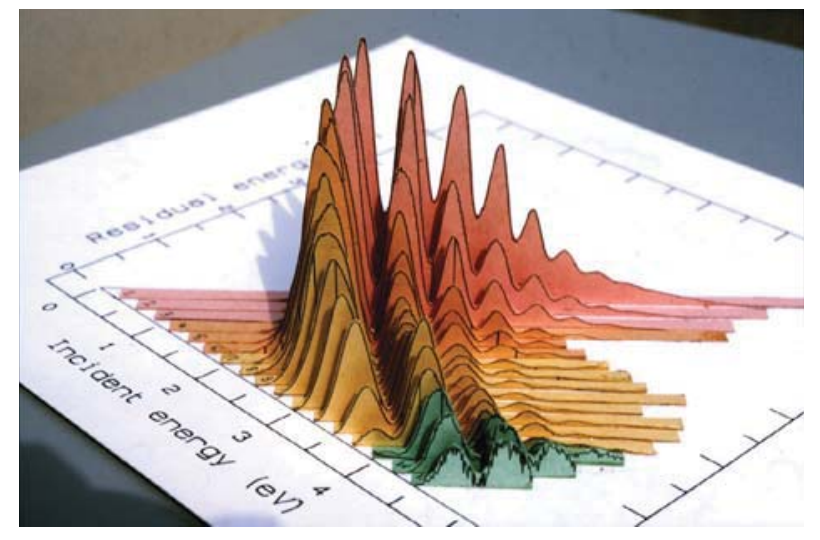

Fig. 7. An early 2-D EELS of nitrogen.

packet "rains down" along its path above the neutral potential surface and the final vibrational states carry (indirectly) information about where it rained down and thus allow us to "spy" on its trajectory. Changing the incident electron energy allows us to choose in which initial vibrational state (or boomerang state when the autodetachment is fast and the state is lifetime broadened) the resonance is prepared, i.e., into what direction (along what normal mode) is the nuclear wave packet initially sent. The spectra of the detached electrons carry information about the packet propagation, they tell us into what mode and into which quanta the wave packet dropped, and whether it changed modes by IVR.

The idea of 2D spectra is not new, 2D electron spectra were used by Reddish et al. in the past to study $\mathrm{N}_{2}$ [18] and by Currell and Comer to study $\mathrm{CO}_{2}$ [19]. Figure 7 shows an early 2D-EEL spectrum of $\mathrm{N}_{2}$, fabricated 1985 in Fribourg from cardboard. It is based on the data from the Fribourg magnetically collimated (trochoidal) electron spectrometer [20]. The present work improves the quality of the spectra and promotes it as a general tool to study nuclear dynamics of resonances.

We applied the technique to acrylonitrile $[21,22]$ and methacrylonitrile [21]. The acrylonitrile spectrum is shown in Figure 8 as an example.

The way to read the spectrum is as follows. The vertical scale is the incident energy and determines the initial (vibrational) state of the resonance. In quantum world this scale determines not only the energy, but also the direction (normal coordinate) into which the cloud of the wave packet is sent. As an example, at an incident energy of just below $0.4 \mathrm{eV}$ the resonance is prepared with one quantum of the $\mathrm{C} \equiv \mathrm{N}$ stretch $\nu_{4}$ excited. The horizontal coordinate indicates the final state, that is, the large dot at an energy loss of about $0.3 \mathrm{eV}$ indicates that our $\nu_{4}$ resonance decays preferentially into $\nu_{4}$ excited neutral molecule. The preferred detachment is thus without change of vibrational quantum and without change of mode - without IVR. (This is, however, not a general rule, cases will be reported in the future where IVR dominates). Processes where the electron leaves with nearly zero energy are located along the "threshold line". 


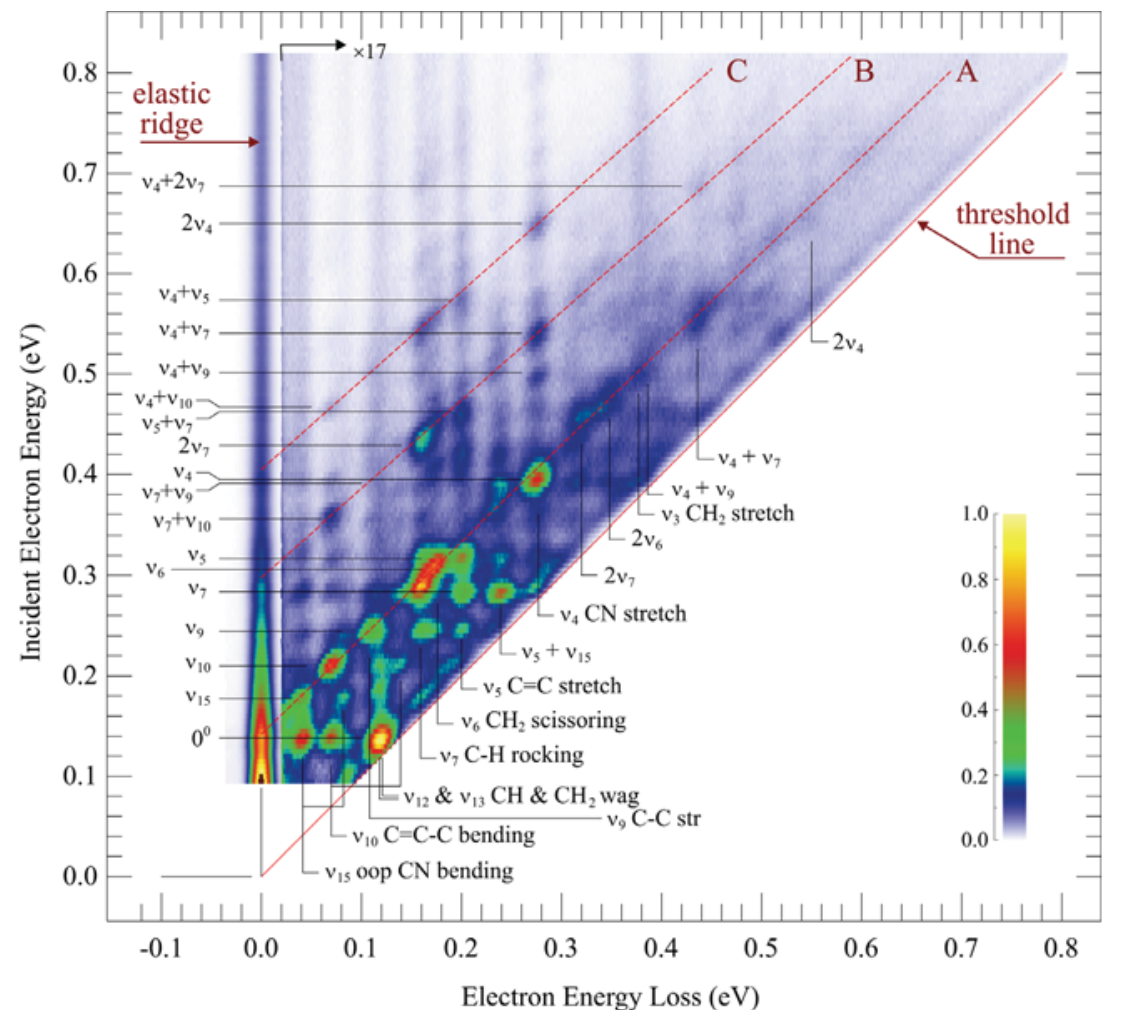

Fig. 8. 2-D EELS of acrylonitrile (from Ref. [21]).

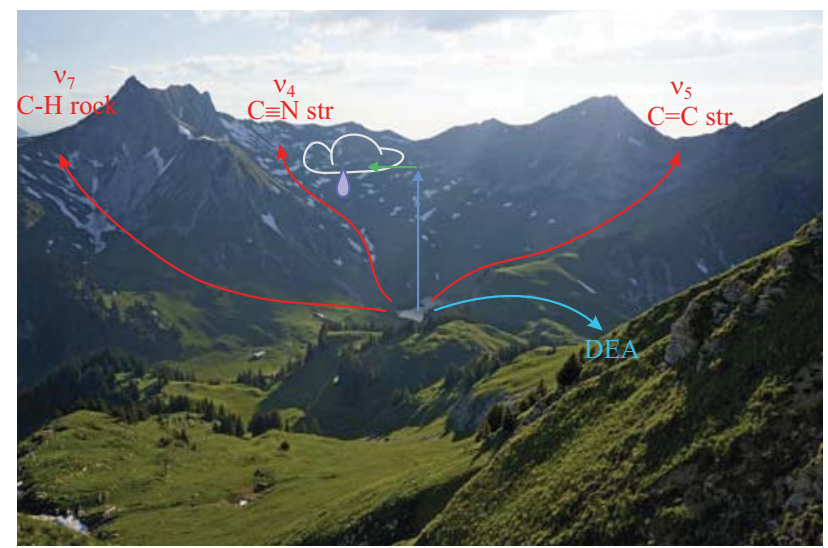

Fig. 9. View of the pre-Alpes in the Canton of Fribourg illustrating the motion of a nuclear wave packet (the cloud) above the landscape of the potential surface of the neutral molecule. Different directions (normal modes) are indicated. Detachment is symbolized by the "cloud" "raining" down whereby the depth by which the "rain drops" fall corresponds to the detached electron energy and is a measurable quantity. The direction to the right leads to a pass below the altitude of the cloud, symbolizing dissociative attachment.

The Fribourg countryside is particularly suitable for contemplating these processes as illustrated in an anecdotal but revealing way in Figure 9. Electron attachment lifts the nuclear wave packet (the "cloud") from the ground vibrational state of the neutral molecule (the lake at the bottom) to a variable altitude determined by the incident electron energy. The cloud is sent to a direction $(\mathrm{C} \equiv \mathrm{N}$ stretch, $\mathrm{C}-\mathrm{H}$ rock or $\mathrm{C}=\mathrm{C}$ stretch in the picture) determined by the vibrational mode to which the incident electron energy corresponds. The cloud "rains down" along its motion, whereby the height by which the drops fall corresponds to the detached electron energy and is a measurable quantity. It indicates how far up the valley did the cloud move before detaching. In Figure 8 this "up valley" motion corresponds to approaching the "threshold line" and the 2D spectrum thus implicitly contains the time variable. At the same time Figure 8 indicates the final vibrational mode, that is, in which valley did it "rain". The obvious case is when it "rains" into the same valley as the one into which it was sent-the vibrational mode is preserved - as in the $\nu_{4} \mathrm{C} \equiv \mathrm{N}$ stretch example mentioned above. In some cases the "cloud" changes direction and "rains" down in a valley different from that into which it was sent - the spectrum then reveals IVR.

An initially unexpected feature are the diagonal patterns in the spectra. One, labeled diagonal "A", corresponds to all processes where vibrational quantum and mode have not changed in the process of detachment. The left-shifted diagonal "B", corresponds to processes further away from the threshold line, the detached electron is faster, by an amount corresponding to the $\mathrm{C}-\mathrm{H}$ rocking vibration $\nu_{7}$. The de-excitation of the vibration $\nu_{7}$ thus promotes electron detachment. Similarly processes along the diagonal "C" were promoted by de-excitation of the vibration $\nu_{4}$.

$2 \mathrm{D}$ spectra of a number of compounds were recently recorded in Fribourg and are being prepared for 


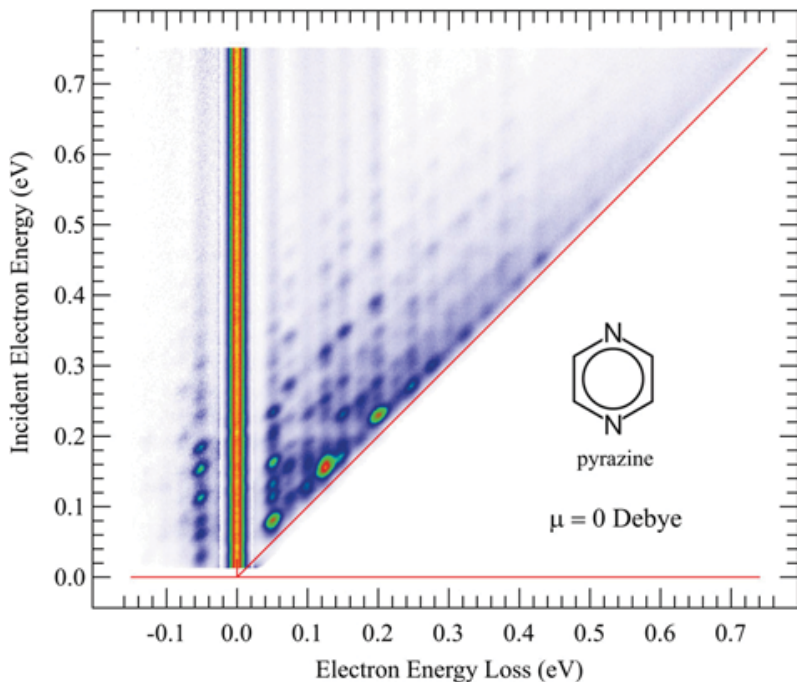

Fig. 10. 2D EELS of pyrazine.

publication. Figure 10 shows a preliminary spectrum of pyrazine. Note that to enhance the visibility of weak features the "elastic ridge" was normalized to one at all energies whereas in Figure 8 the true elastic value is shown. Pyrazine does not have a permanent dipole and the sharp structures are therefore likely due to a $\pi^{*}$ resonance and not to dipole bound state. Diagonal patterns, like in acrylonitrile, prevail.

\section{Electronic excitation of unsaturated compounds}

Pyrimidine serves as a simple model compound for the nucleobases cytosine, thymine and uracil, making electron collisions with pyrimidine relevant for radiation damage to living tissue and thus for cancer radiotherapy [23]. Electronic excitation plays a key role because it is likely to be the initial step in the process of neutral dissociation.

We measured the elastic [24] and the electronically inelastic [25] cross sections of pyrimidine. The cross sections were measured with emphasis on including the threshold region and on recording the shape of the excitation function, i.e., on unraveling the excitation mechanism by detecting resonances in the plot of the cross section as a function of energy. Absolute differential cross sections were combined to obtain integral cross section such as shown in Figure 11. It shows sample data for the lowest energy-loss band, peaking at the energy loss of $4.26 \mathrm{eV}$.

The cross sections were further calculated using $\mathrm{R}$ matrix theory as detailed in reference [25] and the results for the pertinent states are also shown in Figure 11. Note that the experiment and the R-matrix results are shown on their own absolute scales, without renormalization, and that the confidence limit of the experimental data is $\pm 25 \%$ (two standard deviations). Experiment and theory agree within the experimental error bars!

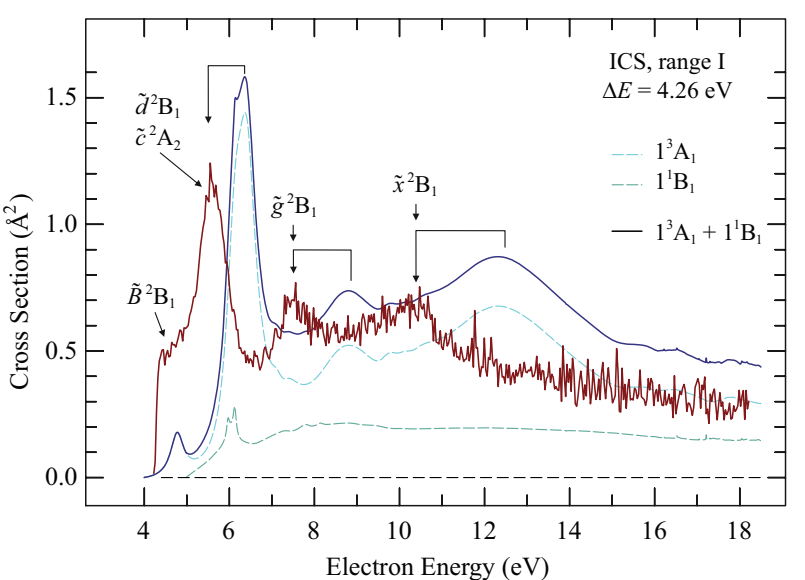

Fig. 11. Red line: integral cross section recorded at an energy loss of $4.26 \mathrm{eV}$ and normalized such as to reflect all excitation processes within the range I, i.e., between $3.52 \mathrm{eV}$ and $4.78 \mathrm{eV}$. The dark blue solid line: the sum of the cross sections calculated for the two states dominating the energy loss of $4.26 \mathrm{eV}$ to which the experimental cross section refers. Dashed lines: the calculated cross sections for these two states shown individually (adopted from Ref. [25]).

Table 1. Selected resonances in pyrimidine. Vertical attachment energies are given. See references [25,26] for more complete lists of the resonances.

\begin{tabular}{cccc}
\hline Label & Exp. & R-matrix & Configuration \\
\hline$\tilde{X}^{2} A_{2}$ & 0.27 & 0.53 & $\pi_{2}^{*}$ shape \\
$\tilde{A}^{2} B_{1}$ & 0.70 & 0.96 & $\pi_{1}^{*}$ shape \\
$\tilde{B}^{2} B_{1}$ & 4.35 & 4.78 & $\pi_{3}^{*}$ shape $+\pi, \pi^{* 2}$ core excited \\
$\tilde{c}^{2} A_{2}$ & 5.55 & 6.11 & $\pi, \pi^{* 2}$ core excited \\
$\tilde{d}^{2} B_{1}$ & 5.55 & 6.37 & $\pi, \pi^{* 2}$ core excited \\
$\tilde{g}^{2} B_{1}$ & 7.45 & 8.47 & - \\
$\tilde{x}^{2} B_{1}$ & 10.3 & 12.3 & - \\
\hline
\end{tabular}

Equally remarkable, experiment and theory agree on the shape of the curve, with the exception that the resonant peaks are consistently calculated shifted to higher energies. The calculation is thus validated by the comparison with the experiment and yields reliable assignment of the resonances, given in part in Table 1, and thus clarifies the excitation mechanism.

The upward shift of the calculated resonances is not a very serious problem in this respect because the reasons for it are well understood. Resonance positions and widths depend sensitively on polarization/correlation effects. They are modeled by including a number of electronically excited target states coupled to the continuum orbitals and by the $L^{2}$ functions in the Close-Coupling expansion [27] - and only a limited number of $L^{2}$ configurations can be included. In addition, our calculated target electronic states tend to appear higher in energy than more accurate calculations and experiments predict. Both facts combine to cause the overestimation of the resonance positions.

The present results fit well the broader picture of nearthreshold excitation of the lowest triplet state in a number 
of unsaturated compounds with ethene, furan, benzene and pyrimidine being the well studied examples [25,28,29]. In all cases, the cross section has a shoulder at or slightly above threshold, due to a nominally shape resonance, which decays into an electronically excited state either because it contains an admixture of core excited configurations or because (in ethene) the shape resonance can be regarded as a parent state of the triplet state, resulting when an electron is formally removed from an occupied orbital. A peak due to a $\pi, \pi^{* 2}$ core excited resonance is found in all cases above the shape resonance shoulder. This peak overlaps with, and is followed by, other coreexcited resonances in molecules with many orbitals like pyrimidine, but the general aspect of the spectra is the same.

\section{Conclusions}

Proof of principle has been provided that electron collisions with surfaces of ionic liquids are an interesting process worth further study. Future work may include study of solvent-solute interactions and solute presence on the surface in dependence on their chemical structure. Chemical transformation of the ionic liquid and the solute induced by the electrons is also of interest. There is also the prospect of fabricating nanodevices by FEBID on the liquid surfaces which could then be moved around or detached by diluting the IL.

Study of dynamics of nuclear motion in resonances offers a fundamental advantage over a similar study in neutral molecules (i.e., in photochemistry) because the detached electrons carry information on the process of relaxation. This information is suitably presented in $2 \mathrm{D}$ spectra and it has been shown here that it is interesting to record such spectra in a systematic way for a series of compounds. Initial work on a wider series of compounds has recently been performed in Fribourg and is being prepared for publication.

Absolute cross sections for electronic excitation of pyrimidine have been recorded with emphasis on the resonant structure in the excitation functions. R-matrix theory was very successful in reproducing both the absolute values and the resonant structure. The theoretical work, validated by the comparison with experiment, provides assignment of the resonances and thus clarifies the mechanism of the excitation. This then reveals common general patterns in electronic excitation of chromophores with double bonds, in particular contributions of $\pi^{*}$ shape resonances near threshold and contributions of $\pi, \pi^{* 2}$ core excited shape resonances at higher energies.

\section{Author contribution statement}

Khrystyna Regeta performed the majority of the measurements, Jimena D. Gorfinkiel and Zdeněk Mašín performed the R-matrix calculations on pyrimidine and did the associated writing, Stefan Grimme and Christoph
Bannwarth calculated the excited states of the ionic liquids and did the associated writing. Michael Allan assisted to the measurements and did most of the writing.

This research is part of Project No. 200020-144367/1 of the Swiss National Science Foundation and of the COST Action CM1301 CELINA. C.B. and S.G. are grateful for support by the Fonds der Chemischen Industrie and the DFG in the framework of the SFB 813 ("Chemistry at Spin-Centers"). Z.M. and J.D.G. acknowledge the support of EPSRC in funding UK-RAMP Project and of the ARCHER eCSE01-013 project; Z.M. acknowledges the use of the VULCAN computer cluster at the Max-Born Institute.

\section{References}

1. M. Allan, J. Phys. B 25, 1559 (1992)

2. M. Allan, J. Phys. B 40, 3531 (2007)

3. M. Allan, Phys. Rev. A 81, 042706 (2010)

4. E. Harting, F.H. Read, Electrostatic Lenses (Elsevier Science Ltd, London, 1976)

5. M. Allan, Phys. Scr. T 110, 161 (2004)

6. F.H. Read, J.M. Channing, Rev. Sci. Instrum. 67, 2373 (1996)

7. M. Zubek, N. Gulley, G.C. King, F.H. Read, J. Phys. B 29 , L239 (1996)

8. F.H. Read, N.J. Bowring, Charged particle optics program, CPO-3D, ww. electronoptics.com

9. M. Allan, J. Phys. B 38, 3655 (2005)

10. K. Regeta, C. Bannwarth, S. Grimme, M. Allan, Phys. Chem. Chem. Phys. 17, 15771 (2015)

11. B. Kučera, Drud. Ann. Phys. 11, 529 (1903)

12. J. Heyrovský, Trans. Faraday Soc. 19, 692 (1924)

13. T. Hamann, L. Kankate, E. Böhler, J.H. Bredehöft, F.M. Zhang, A. Gölzhäuser, P. Swiderek, Langmuir 28, 367 (2012)

14. T. Goulet, J.P. Jay-Gerin, Radiat. Phys. Chem. 27, 229 (1986)

15. M. Allan, J. Electron Spectrosc. Relat. Phenom. 48, 219 (1989)

16. S. Grimme, W. Hujo, B. Kirchner, Phys. Chem. Chem. Phys. 14, 4875 (2012)

17. A. Dreuw, J.L. Weisman, M. Head-Gordon, J. Chem. Phys. 119, 2943 (2003)

18. T. Reddish, F. Currell, J. Comer, J. Phys. E 21, 203 (1988)

19. F. Currell, J. Comer, Phys. Rev. Lett. 74, 1319 (1995)

20. M. Allan, J. Phys. B 18, 4511 (1985)

21. K. Regeta, M. Allan, J. Chem. Phys. 142, 184307 (2015)

22. K. Regeta, M. Allan, Phys. Rev. Lett. 110, 203201 (2013)

23. B. Boudaiffa, P. Cloutier, D. Hunting, M.A. Huels, L. Sanche, Science 287, 1658 (2000)

24. K. Regeta, M. Allan, C. Winstead, V. McKoy, Z. Mašín, J.D. Gorfinkiel, J. Chem. Phys. 144, 024301 (2016)

25. K. Regeta, M. Allan, C. Winstead, V. McKoy, Z. Mašín, J.D. Gorfinkiel, J. Chem. Phys. 144, 024302 (2016)

26. Z. Mašín, Ph.D. thesis, The Open University, Milton Keynes, UK, 2012

27. Z. Mašín, J.D. Gorfinkiel, J. Chem. Phys. 137, 204312 (2012)

28. M. Allan, C. Winstead, V. McKoy, Phys. Rev. A 77, 042715 (2008)

29. K. Regeta, M. Allan, Phys. Rev. A 91, 012707 (2015) 